38. U.S. Office of Management and Budget (OMB), Circular A-4 (OMB, Washingtion, DC, 2003).

39. D. B. Jelliffe, Nutr. Rev. 30, 199 (1972).

40. WHO, International Code of Marketing of Breast-milk Substitutes (WHO, Geneva, 1981).

41. ]. P. Brady, Arch. Dis. Child. 97, 529 (2012).

42. WHO, UNICEF, Academy for Educational Development USAID, Africa's Health in 2010. Learning from Large-Scale Community-Based Programmes to Improve Breastfeeding Practices (WHO, Geneva, 2008).

43. S. Horton et al., Health Policy Plan. 11, 156 (1996).
44. D. ]. Chapman, K. Morel, A. K. Anderson, G. Damio, R. Pérez-Escamilla, J. Hum. Lact. 26, 314 (2010).

45. R. T. Lester et al., Lancet 376, 1838 (2010).

46. C. K. Lutter, C. M. Chaparro, L. M. Grummer-Strawn, C. G. Victora, Am. J. Public Health 101, 2130 (2011).

47. M. F. Rea, Caude Salud Pub. 19, 119 (2003).

48. C. K. Lutter et al., Pediatrics 128, e1418 (2011).

49. K. Edmond, R. Bahl, Optimal Feeding of Low-Birth-Weight Infants (WHO, Geneva, 2006).

50. L. C. Mullany et al., J. Nutr. 138, 599 (2008).
51. WHO and partners, Indicators for Assessing Infant and Young Child Feeding Practices. Part 3: Country Profiles (WHO, Geneva, 2010).

52. J. G. Woo et al., J. Pediatr. Gastroenterol. Nutr. 54, 532 (2012)

Acknowledgments: We thank the unnamed reviewers and C. Monteiro for their constructive and expert comments on an earlier draft of this paper. No outside funding was received for this paper, and we declare no conflicts of interest.

$10.1126 /$ science. 1224616

\title{
Double Burden of Noncommunicable and Infectious Diseases in Developing Countries
}

\section{C. Bygbjerg}

On top of the unfinished agenda of infectious diseases in low- and middle-income countries, development, industrialization, urbanization, investment, and aging are drivers of an epidemic of noncommunicable diseases (NCDs). Malnutrition and infection in early life increase the risk of chronic NCDs in later life, and in adult life, combinations of major NCDs and infections, such as diabetes and tuberculosis, can interact adversely. Because intervention against either health problem will affect the other, intervening jointly against noncommunicable and infectious diseases, rather than competing for limited funds, is an important policy consideration requiring new thinking and approaches.

$\mathrm{I}$ n 1971, Omran (1) described how health and disease patterns change over time in societies, depending, among other factors, on the degree of demographic transition and rate of economic development, to result in an epidemiological transition. Like individuals, societies have a "life cycle": In a "young" society, infectious diseases and nutritional deficiencies dominate; hence in children, diarrhea and acute respiratory infections, including measles and malaria, predominate; in pregnant women, fetal loss, perinatal death from undernutrition, bleeding, and infection are major risks, and in the surviving adults, tuberculosis (TB) and other diseases related to poverty are important causes of morbidity. When societies "grow up," accidents and violence-related disabilities and deaths increase, mostly among the young, and although infectious diseases such as TB still prevail, chronic noncommunicable diseases (NCDs) become more prevalent, particularly in urban populations. In aging societies, NCDs predominate: first, type 2 diabetes and cardiovascular diseases, followed by cancer and degenerative disorders. Simultaneously, in extreme cases the broad-based demographic pyramid inverts.

Demographic transition as the main explanation for the growing NCD burden has, however, been questioned. Stuckler (2), in a thorough analysis of

Copenhagen School of Global Health, Department of International Health, Immunology and Microbiology, Faculty of Health Sciences, University of Copenhagen, 5 Øster Farimagsgade, DK-1014, Copenhagen K, Denmark. E-mail: iby@sund.ku.dk.

causes that was published in the same journal as Omran's historical paper, pointed out that particularly in low-income countries, economic growth, market integration, foreign direct investment, and urbanization together correlated threefold greater to epidemiological transition than did population aging. The projections of disease burden in Fig. 1 are made by considering the combined effect of demographical transition (population growth and increasing life expectancies) and expected impact of changing lifestyle, living conditions, and economic development (3).

Omran (1) has also been criticized for overlooking new epidemics of infectious diseases, but this author could not have predicted the HIV epidemic, which disturbed his model, set back the epidemiological and demographic transition, and, more importantly, reversed the reduction of deaths from infections in children and young adults, particularly in subSaharan Africa. As a consequence, combating HIV and other major infections and improving child and maternal health remained prominent among the Millennium Development Goals (MDGs) set in 2000. Similarly, the United Nations' General Assembly (UNGASS) in 2001 committed all governments to combat the HIV epidemic (but not NCDs), and, consequently, WHO and UNAIDS updated their "Strategies for the Prevention and Control of Communicable Diseases" (4). In that document, $\mathrm{NCDs}$ - such as diabetes, as a potential risk factor for infections, or TB in particular-were not mentioned. None of the MDGs relate directly to NCDs, although Stuckler et al. (5) and others have indicated that MDGs may not be attained without addressing NCDs as risk or cofactors for communicable diseases.

Before the turn of the millennium, some researchers $(6)$ and the World Health Organization (WHO) (7) had pointed at the danger of a "double burden of disease," such as the emerging epidemic of chronic NCDs, in addition to the "unfinished agenda of infectious diseases" and problems of maternal and child health. Yach et al. (8) showed that even in the poorest countries, more deaths are caused by NCDs than from infections, and that the WHO Headquarters spent only US $\$ 0.50$ on chronic diseases per death per person compared with US\$7.50 for leading communicable diseases. Yet in 2005, the WHO in its report "Preventing chronic diseases - A vital investment" ( 9 ) underscored that NCDs do not only hit the old, the rich, and the fat; developing countries carry the heaviest burden of NCDs. In 2007, the World Bank (WB) issued a similar report on the conceptions, misconceptions, and challenges presented by chronic NCDs (10). In 2011, partly as a result of these reports and provoked by continuous lobbying by civil society and leading stakeholders in NCDs, including the International Union Against Cancer, the World Heart Federation, the Global Alliance against Chronic Respiratory Diseases, the International Diabetes Federation, and the International Union Against Tuberculosis and Lung Disease, UNGASS committed governments to fight the emerging epidemic of NCDs, acknowledging that NCDs hit developing countries hard (11). When reading and comparing the UNGASS declarations from 2001 and 2011, unfortunately, the known and potential links between infectious diseases and NCDs are barely visible. Similarly, in the 182-page 2005 WHO report (9) and the 188-page $2007 \mathrm{WB}$ report (10) on NCDs, TB is mentioned once in each report, malaria once in the WHO report, and HIV six and three times, respectively.

A major barrier for integrated intervention against the double burden of infections and NCDs may be that their etiologies and pathologies at first glance appear to be diametrically opposed. As part of new public management, researchers, health professionals, donor agencies, and politicians are often forced to focus on a particular health problem to get visible results and fulfill result contracts. At a time of global financial crises and shrinking health budgets, there is a threat that the battle against common 
and future health problems, both communicable and noncommunicable, may become a fight for funds to control either health problem, rather than a fight against the double burden of disease.

A key question to be answered before setting priorities and allocating (or reallocating) resources for health beyond 2015 is whether the sharp demarcation between communicable diseases and NCDs, which is apparent in most projections of global mortality and burden of disease - including WHO's in Fig. 1-is justified when both may hit the same individuals and societies. In the following, I highlight some potential and verified links between diseases and discuss possible ways of addressing them jointly and/or without unnecessary competition for financial or human resources.

Intensifying interventions against joint risk factors for NCDs - including diabetes, chronic obstructive pulmonary disease, some cancers, and against a major infectious disease, TB - by fighting malnutrition (12) and tobacco and alcohol use is an obvious priority. Preventing common cancers by vaccinating against the virus that induces them, including human papilloma virus (HPV) and hepatitis $\mathrm{B}(\mathrm{HBV})$, is another priority.

If NCDs could be prevented by improving maternal and child health, it would be welcomed by policy- and decision-makers in low- and middleincome countries (LMICs). But how much are NCDs in adults related to ill-health in mothers and children? Indeed, infection and deficiencies in pregnancy, such as malaria and anemia, often result in fetal and maternal loss, prematurity, and low birth weight (13), and it is established that undernutrition and communicable diseases in childhood do interact (14). It is less well known that adverse events in early life play a major role in cardiovascular diseases and diabetes. In the 1980s, Barker and Osmond (15) found that ischemic heart disease mortality rates strongly correlated with neonatal and postneonatal mortality 50 years earlier. This prompted Hales and Barker's "thrifty phenotype theory," now confirmed, that low birth weight may induce early debut of cardiovascular disease and type 2 diabetes (16), as also described by Lutter and Lutter in this issue (17). Thus, intensifying programs to improve maternal and child health could reduce risks for NCDs. As we have proposed, and presently are investigating in East Africa, metabolic disease in adult life may even be prevented by malaria control in pregnancy (18). Barker and Osmond (15) showed that poor nutrition in early life increases susceptibility to the effects of a more affluent diet in later life. In many LMICs, a nutritional transition may take place in a single generation, and it is possible that induction of insulin resistance by epigenetic silencing of insulin-regulating genes in utero could be the mechanism (19). Insulin resistance induced in early life may be reversible, but if catchup growth is too fast, some data point at an increased risk of low-birth-weight children becoming obese (20). Breast-feeding is strongly recommended for low-, as well as normal-, birth-weight and large (macrosomic) babies and protects against diarrhea, respiratory infections, and obesity (21). Overweight mothers and high-birth-weight neonates are at high risk for developing diabetes (22). Promotion of breast feeding would be a low-cost, low-hanging tool for the prevention of NCDs, as well as infection (17).

Screening for gestational diabetes could be part of an integrated, antenatal care program, along with screening for and prevention of malaria, HIV, and HBV transmission from mother to child. HIV increases the risk for HPV-induced cervical cancer threefold, and $80 \%$ of cervical cancer cases occur in LMICs (23). Presently, highly effective but costly vaccination against HPV is mainly available in highincome countries, although vaccination against HBV is increasingly becoming part of expanded programs on immunization, even in LMICs, preventing cirrhosis, as well as cancer of the liver.

The HIV epidemic taught us that the risk for developing TB increases more than fourfold where both diseases are prevalent. In spite of this, it took almost 20 years before the WHO and UNAIDS managed to agree on guidelines for the implementation of collaborative TB and HIV program activities (24). HIV plus malaria also negatively affect human health; likewise, it took 20 years to formulate recommendations on how to fight them jointly (25). Hopefully, strategies for combating the double burden of communicable diseases and NCDs have a shorter incubation time. There are some success stories.

Diabetes' interaction with TB was recognized early but later forgotten by clinicians and public health experts when TB declined in the Western world. Only when diabetes rose exponentially in TB-prevalent LMICs were interactions and implications for control reviewed (26). This review and other publications documented that the risk for developing TB increases threefold in diabetics, and that TB may in turn increase the risk for type 2 diabetes by inducing glucose intolerance and deteriorating glycemic control (27). Fortunately, the lessons learned from the HIV-associated TB prompted a much faster reaction to the looming epidemic of diabetes-associated TB (28). A framework has been established in less than 5 years to guide national programs, clinicians, and others engaged in care of patients on how to establish a coordinated response to both diseases at organizational and clinical levels. In China, India, and other countries with high burdens of both diseases, large-scale programs for dual screening and management are now being rolled out (29). One example of applying management tools from TB directly to the management of diabetes in a low-income country is the successful introduction of "DOTS for Diabetes" in Malawi (30).

As Halfdan Mahler, "the father of primary health care" stressed back in 1966 (31), when attempting to convince decision-makers that a double burden of disease requires integrated control strategies that should begin in the primary health care sector (32), it should not be forgotten that "integration, far from being a laissez-faire approach, requires maximum involvement of all specialized personnel such as

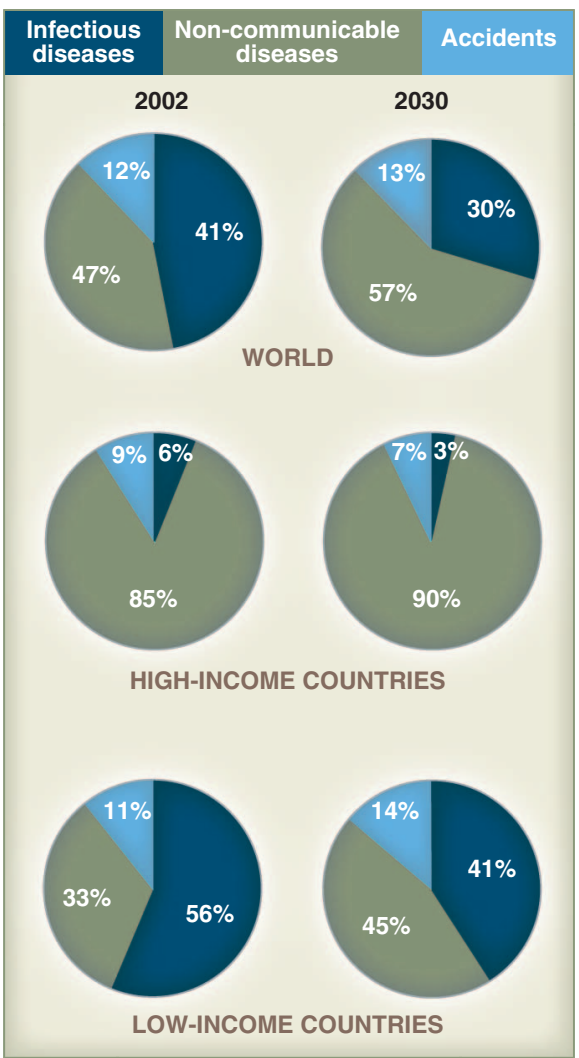

Fig. 1. The proportional distribution of disabilityadjusted life years, contributable to infectious diseases and NCDs for (top) the world, (middle) high-income countries, and (bottom) low-income countries for 2002 and 2030 (3).

programmers, organizers, tutors and assessors." Thus, when adjusting health systems to meet the challenges of the double burden of disease, whether caused by aging or changing living conditions or both, a multidimensional approach is needed, ranging from education, equity, and economic development to environmental change (33).

\section{References and Notes}

1. A. B. Omran, Milbank Q. 49, 509 (1971).

2. D. Stuckler, Milbank Q. 86, 273 (2008).

3. C. D. Mathers, D. Loncar, Updated projections of global mortality and burden of disease, 2002-2030. World Health Organization; available at www.who.int/ healthinfo/global_burden_disease/en/index.html.

4. WHO, UNAIDS, Strategies for the Prevention and Control of Communicable Diseases (WHO/CDS/CPE/SMT/ 2001.13)

5. D. Stuckler, S. Basu, M. McKee, PLoS Med. 7, e1000241 (2010).

6. C. J. L. Murray, A. D. Lopez, Eds., Global Burden of Disease and Injury Series, vol. I (Harvard School of Public Health on behalf of the World Health Organization and the World Bank, Cambridge, 1996).

7. WHO, The Double Burden: Emerging Epidemics and Persistent Problems. The World Health Report 1999 (WHO, Geneva, 1999).

8. D. Yach, C. Hawkes, C. L. Gould, K. J. Hofman, JAMA 291 2616 (2004).

9. WHO, Preventing Chronic Diseases-A Vital Investment, WHO Global Report (WHO, Geneva, 2005). 
10. The World Bank, Public Policy and the Challenge of Chronic Noncommunicable Diseases (The World Bank, Washington, DC, 2007).

11. WHO, United Nations high-level meeting on noncommunicable disease prevention and control. NCD summit to shape the international agenda, September 2011; available at www.who.int/nmh/events/ un ncd summit2011/en/.

12. C. Dye, B. Bourdin Trunz, K. Lönnroth, G. Roglic, B. G. Williams, PLOS ONE 6, e21161 (2011).

13. I. A. McGregor, M. E. Wilson, W. Z. Billewicz, Trans. $R$. Soc. Trop. Med. Hyg. 77, 232 (1983).

14. I. Philips, B. Wharton, BMJ 5589, 407 (1968).

15. D. J. P. Barker, C. Osmond, Lancet 327, 1077 (1986).

16. C. N. Hales et al., BMJ 303, 1019 (1991).

17. C. K. Lutter, R. Lutter, Science 337, 1495 (2012).
18. D. L. Christensen, A. Kapur, I. C. Bygbjerg, Int. J. Gynaecol. Obstet. 115, (suppl. 1), S16 (2011)

19. C. ]. Nolan, P. Damm, M. Prentki, Lancet 378, 169 (2011).

20. C. Lau, ]. M. Rogers, M. Desai, M. G. Ross, Obstet. Gynecol. 117, 978 (2011).

21. G. V. Agrasada, U. Ewald, E. Kylberg, ]. Gustafsson, Asia Pac. J. Clin. Nutr. 20, 62 (2011).

22. E. L. Leikin, J. H. Jenkins, G. A. Pomerantz, L. Klein, Obstet. Gynecol. 69, 570 (1987).

23. M. Dartell et al., Sex. Transm. Dis. 39, 201 (2012).

24. WHO, Guidelines for Implementing Collaborative TB and HIV Programme Activities (WHO/CDS/TB/2003.319).

25. WHO, Malaria and HIV/AIDS Interactions and Implications; available at www.who.int/malaria/ publications/atoz/9241593350/en/index.html.

26. C. Y., PLoS Med. 15, e152 (2008)
27. K. E. Dooley, R. E. Chaisson, Lancet Infect. Dis. 9, 737 (2009).

28. A. D. Harries et al., Int. J. Tuberc. Lung Dis. 15, 1436, i (2011)

29. WHO, Collaborative Framework for Care and Control of Tuberculosis and Diabetes (WHO/HTM/TB/2011.15); available at www.who.int/diabetes/publications/ tb_diabetes2011/en/index.html.

30. T. J. Allain et al., Trop. Med. Int. Health 16, 1077 (2011)

31. M. Uplekar, M. C. Raviglione, Bull. World Health Organ. 85, 413 (2007).

32. L. Li et al., Trop. Med. Int. Health., published online 25 July $2012(10.1111 / j .13652012 .03068 . x)$

33. I. C. Bygbjerg, Ugeskr. Laeger 174, 1516 (2012) [Challenges and solutions in global health (Danish)].

10.1126/science. 1223466

\title{
Why a Macroeconomic Perspective Is Critical to the Prevention of Noncommunicable Disease
}

\author{
Richard Smith
}

Effective prevention of noncommunicable diseases will require changes in how we live, and thereby effect important economic changes across populations, sectors, and countries. What we do not know is which populations, sectors, or countries will be positively or negatively affected by such changes, nor by how much. Without this information we cannot know which policies will produce effects that are beneficial both for economies and for health.

B ill Shankly (manager of Liverpool Football Club from 1959 to 1974) said football (soccer) is "not just a matter of life and death, it's more important than that." For economists, so are noncommunicable diseases (NCDs) (1). Not only are the effects of NCDs felt throughout the economy (Table 1), but since the agents contributing to NCDs are influenced by our lifestyles, effective preventive policies are likely to include mechanisms that themselves have appreciable economic impacts, such as taxing soft-drinks, increasing the use of public transport, or promoting lower-polluting energy sources $(2,3)$. Although the impacts of such policies may improve health, there will be substantive economic impacts as they ripple out through the economy, generating differential effects across various sectors, such as housing, transport, and agriculture. These economic effects may generate yet further health effects, which themselves then feed into the economy, generating yet more cycles of effects. This interaction and reciprocity between NCDs and the economy highlights the critical need for a macroeconomic perspective in the design, implementation, and evaluation of preventive policies to tackle NCDs.

Health System Economics, Faculty of Public Health and Policy, London School of Hygiene and Tropical Medicine, 15-17 Tavistock Place, London WC1H 9SH, UK. E-mail: richard.smith@lshtm. ac.uk
Macroeconomics, as compared with microeconomics (which is focused upon "partial equilibrium" within a single sector, such as for housing or meat), is concerned with general equilibrium across all sectors, and thus how changes in one sector (e.g., increase in price) affect other sectors, with all these changes together comprising the overall "economic impact" of a single change $(4,5)$. For instance, the impact of pandemic influenza on the healthcare sector is minimal compared with its effect on gross domestic product (GDP) through impacts on other sectors (e.g., hotels, leisure, travel), which are a consequence of changes in individual behavior in response to pandemic threat and the mitigation policies themselves $(6,7)$.

\section{Why Is This Important for NCD Prevention?}

NCDs, such as diabetes, cancer, and heart disease, differ from infectious diseases, such as pandemic influenza, as they are not transmitted from person to person [although there is evidence emerging in the social sciences of "social contagion," where social networks appear to influence the probability of obesity, for instance (8)]. However, they also differ in that they are intrinsically lifestyle diseases, and hence the cause and impact are linked in a multiplicity of ways to everyday economic activity (Fig. 1).

NCD-related health (Fig. 1, box 1) is determined directly by risk factors (Fig. 1, box 2), which include genetic predisposition to disease, such as diabetes and heart disease, but also by a range of other social determinants of health, which refer to the general conditions in which people live and work, including levels and types of employment, environmental conditions, and education ( 9 ). These social determinants, contribute to the risk of different diseases, such as pollution-related diseases and cancer. They are also intimately linked with the household and individual (Fig. 1, box 3), which represent how people behave and, crucially, invest (or disinvest) in their health by what they consume and in the activities they undertake (8). For example, cancer and heart disease risk will be affected by decisions concerning smoking, alcohol consumption, and exercise. But risk will also influence household and individual behavior. For instance, an individual's knowing that they have a higher genetic risk of heart disease may modify individual consumption of fast food. The healthcare sector (Fig. 1, box 4) comprises goods and services consumed by households principally to improve health status. Although these affect NCD-related health directly, they also impact on the household economy, which ultimately pays for them through taxation, insurance, or out-of-pocket. The level of ill-health caused by NCDs will also feed back and impact on the household, thus further affecting the risk of other health problems through reducing household income, and feed into healthcare provision through shaping the demand for services, and hence profile of provision (e.g., more insulin prescriptions).

Activity in all non-healthcare sectors in the economy (Fig. 1, box 5), such as agriculture, manufacturing, and education, impacts on the previous three components and, thus, NCDs. It is well established, for instance, that "wealthier is healthier" $(10,11)$, but that wealth also brings an increase in NCD risk, such as through changes in dietary habits, with the suggestion that in some cases this means that economic recessions can have positive health benefits (12). As countries grow wealthier, their populations experience increased desirability and availability of processed foods, perhaps mostly starkly indicated by the experience of some Pacific island populations where traditional diets have been displaced with high-fat imported foodstuffs and a concomitant increase in obesity rates and NCDs. Similarly, the transformation of food retail as countries become 